La communication des neurosciences au tribunal : un courant de recherche à développer [Communicating neurosciences in court: A line of research to develop]

Philippe Leclerc, Véronique Maheux-Caron, Marjorie Grandbois, et Justine Cinq-Mars

Département de psychologie, Université du Québec à

Trois-Rivières, Trois-Rivières, QC, Canada

\title{
Note des auteurs
}

Philippe Leclerc (https://orcid.org/0000-0003-2609-9275)

Véronique Maheux-Caron (https://orcid.org/0000-0001-5553-0090)

Marjorie Grandbois (https://orcid.org/0000-0002-9687-7100)

Justine Cinq-Mars 
Les auteurs ne déclarent aucun conflit d'intérêts. Merci à Anaïs Welsh, avocate, pour ses commentaires et sa révision des aspects juridiques du document.

Toute correspondance portant sur cet article devrait être adressée à Philippe Leclerc. Courriel : philippe.leclerc@uqtr.ca

Soumis : 21 mai 2020

Révision soumise : 28 novembre 2020

Accepté : 14 décembre 2020

(C) 2020, American Psychological Association. This paper is not the copy of record and may not exactly replicate the final, authoritative version of the article. Please do not copy or cite without authors' permission. The final article will be available, upon publication, via its DOI: $10.1037 /$ cap0000278 


\section{LA COMMUNICATION DES NEUROSCIENCES AU TRIBUNAL}

\section{Résumé}

Les neuroscientifiques, cliniciens ou chercheurs, sont de plus en plus sollicités à titre d'experts devant les tribunaux, notamment au Canada. En effet, le tribunal demande parfois à être éclairé sur des éléments permettant de conclure, par exemple, sur la responsabilité criminelle ou sur la peine à imposer chez un accusé en fonction d'une cause reliée au système nerveux. Malgré le développement rapide des neurosciences, le processus de communication au tribunal de cette science n'a pas fait l'objet de la même attention empirique. Cet article vise à souligner les défis du transfert des connaissances, notamment ceux propres au contexte canadien, puis à encourager le développement d'un courant de recherche portant sur la communication des neurosciences par les experts faisant des témoignages au tribunal. Un argumentaire qui justifie la pertinence de développer un tel courant de recherche est présenté. Éventuellement, les découvertes scientifiques dégagées par ce courant de recherche permettront de développer des lignes directrices spécifiques, de parfaire les pratiques des experts, de maintenir leur crédibilité et ultimement d'optimiser le transfert des connaissances. Enfin, un ensemble de devis qualitatifs et quantitatifs sont suggérés.

Mots-clés : communication; neurosciences; tribunal; témoin expert; transfert des connaissances.

\section{Déclaration d'intérêt public}

Les neuroscientifiques, cliniciens ou chercheurs spécialistes du système nerveux, sont de plus en plus souvent sollicités par les avocats afin de témoigner à titre d'experts au tribunal. Toutefois, le processus de communication au tribunal de cette science n'a pas fait l'objet de la même attention empirique. Cet article présente un argumentaire soulignant l'importance d'approfondir la 
LA COMMUNICATION DES NEUROSCIENCES AU TRIBUNAL

recherche sur la communication des neurosciences au tribunal, afin d'assurer un transfert optimal des connaissances. 


\title{
LA COMMUNICATION DES NEUROSCIENCES AU TRIBUNAL
}

\begin{abstract}
Neuroscientists, clinicians and researchers alike, are increasingly called upon to act as expert witnesses in court, including in Canada. Indeed, the court asks to be enlightened to rule on, for instance, the criminal responsibility of an individual or the sentence to give based on a nervous system-related cause. Even if neurosciences are a burgeoning field, communication processes involved in neurosciences expert testimonies have not been the object of the same empirical scrutiny. This article presents pitfalls in knowledge transfer, notably those of the Canadian context, and aims at encouraging the development of a line of research pertaining to the communication of neurosciences by expert witnesses in court. Several arguments are presented. Eventually, scientific discoveries unearthed by this line of research will help to develop specific guidelines, to refine experts' practices, to maintain their credibility in court, and to optimize knowledge transfer. Some qualitative and quantitative research designs are suggested. Keywords: communication; neurosciences, court; expert testimony; knowledge transfer.

\section{Public Significance Statement}

Neuroscientists, i.e., clinicians or researchers who are nervous system specialists, are increasingly called on by jurists to act as expert witnesses in court. However, communication processes involved in neurosciences expert testimonies have not been the object of the same empirical scrutiny. This article presents arguments that highlight the importance to do research about neurosciences communication in court to ensure optimal knowledge transfer. 


\section{La communication des neurosciences au tribunal :}

\section{un courant de recherche à développer}

Les neurosciences se développent rapidement et sont davantage invoquées à titre de

preuve expertale devant les tribunaux (Chandler, 2016). En effet, l'opinion d'un professionnel ou d'un chercheur en neurosciences peut être sollicitée dans un cadre d'expertise légale afin de faciliter l'explication du comportement humain. L'expertise neuroscientifique représente un espoir de déterminer de manière objective la nature, la cause ou l'ampleur d'une particularité liée au système nerveux (p. ex., lésion cérébrale). Ceci permet de répondre à diverses questions d'ordre juridique pertinentes pour le droit civil ou criminel, par exemple en établissant un lien avec cette particularité et les aptitudes parentales ou le comportement criminel. Le témoignage de l'expert peut ainsi jouer un rôle crucial dans le jugement rendu (p. ex., responsabilité criminelle, durée de la peine; Chandler, 2016; Jones, Marois, et al., 2013).

Toutefois, malgré l'étendue des connaissances des experts de ce domaine, l'utilisation des neurosciences comporte plusieurs défis depuis de nombreuses années, notamment en ce qui a trait au transfert des connaissances (p. ex., Illes et al., 2010). Cela devient particulièrement préoccupant lorsque des conséquences d'ordre juridique sont en jeu. Il existe de nombreux défis à traduire les connaissances issues des neurosciences en information accessible, prudente et utile juridiquement, et ce processus communicationnel ne semble pas avoir fait l'objet de la même attention empirique dans le contexte d'expertise légale. Ces constats soulignent l'importance de développer un courant de recherche portant sur la communication des neurosciences au tribunal. Considérant l'impact déterminant que l'expertise peut avoir au tribunal, il est important de s'attarder à comment la forme prise par le témoignage (p. ex., choix de mots, habiletés de vulgarisation, présentation d'images) permet de mieux apprécier la valeur probante réelle de 


\section{LA COMMUNICATION DES NEUROSCIENCES AU TRIBUNAL}

l'expertise (faits scientifiques nuancés). En effet, le transfert des connaissances scientifiques au tribunal est susceptible d'avoir un impact sur la réception du message, son interprétation, son utilisation, et ainsi, sur l'issue du procès. En ce sens, cet article comporte plusieurs volets : (a) une brève description de l'expertise et du rôle du témoin expert, (b) une brève description de l'expertise en matière de neurosciences, (c) les défis du transfert des connaissances issues des neurosciences au tribunal, (d) une illustration issue de la médecine légale des enjeux du témoignage, (e) un argumentaire justifiant la pertinence de développer un courant de recherche sur la communication des neurosciences au tribunal, puis (f) les implications et des directions futures pour les chercheurs.

\section{Expertise légale : concepts de base}

L’expertise légale consiste en un éclairage apporté au tribunal par une personne ayant des connaissances de pointe dépassant celles du juge ou du jury, typiquement d'ordre technique ou scientifique (Barreau du Québec, 2017; Direction des enquêtes du Collège des médecins du Québec, 2016). L'expertise légale se développe autour d'un mandat, clair et précis, dont l'expert a le devoir de s'acquitter de manière impartiale, indépendamment de la partie qui le convoque (Barreau du Québec, 2017; Service des poursuites pénales du Canada, 2011). Il existe deux principaux types de mandats, soit le mandat d'évaluation (expertise sur la personne), visant à se prononcer sur les faits et à donner son opinion professionnelle, et le mandat théorique (expertise théorique), visant à informer le tribunal pour faire état de la connaissance (Brunet, 2014). Le premier mandat implique nécessairement qu'un clinicien membre d'un ordre professionnel (p. ex., neurologue, neuropsychologue) rencontre une personne pour l'évaluer et se prononcer sur sa condition. Le second mandat implique qu'un expert ayant des connaissances, clinicien ou non (p. ex., chercheur), explique des concepts complexes sans faire de recommandations ni de passage 


\section{LA COMMUNICATION DES NEUROSCIENCES AU TRIBUNAL}

du cas général au cas spécifique (c.-à-d., sans appliquer les concepts en question à une des parties; Brunet, 2014; Bureau du syndic et Comité d'inspection professionnelle de l'Ordre des psychologues du Québec, 2002).

L’admissibilité de la preuve expertale au tribunal est encadrée en Amérique du Nord par un certain nombre de critères juridiques définis par l'arrêt Daubert aux États-Unis (Daubert v. Merrell Dow Pharmaceuticals, Inc., 1993) et par l'arrêt Mohan au Canada (R. v. Mohan, 1994; pour plus de détails, voir Stettler, 2019). Ces arrêts ont été rendus afin de remettre entre les mains des juges le rôle de « modérateurs » ou de « gardiens » de l'entrée de la preuve scientifique au tribunal (Stettler, 2019). Les juges doivent se prononcer sur la méthodologie employée ou sur le raisonnement scientifique invoqué afin d'apprécier la robustesse des faits en se basant sur divers critères (p. ex., caractère consensuel des faits au sein de la communauté scientifique, nombre de publications scientifiques, marge d'erreur de la technique employée, etc.). Ultimement, ce processus de filtrage permet aux juges de déterminer l'admissibilité d'un fait scientifique au tribunal (Stettler, 2019). Toutefois, l'opinion de l'expert n'a en aucun cas préséance sur celles des juges; ces derniers ayant toujours le dernier mot en ce qui a trait à l'admissibilité et au poids d'une preuve (Barreau du Québec, 2017).

\section{Expertise en matière de neurosciences}

Au cours des dix dernières années, l'intérêt grandissant accordé aux neurosciences a mené à la création d'un nouveau champ de savoir : le neurodroit (neurolaw; p. ex., Jones \& Shen, 2012). Il s'agit d'une science consacrée à examiner l'impact des découvertes neuroscientifiques dans la procédure judiciaire, notamment d'observer le lien entre le comportement criminel et le dysfonctionnement du cerveau (Jones \& Shen, 2012). Concrètement, dans le cas des expertises sur la personne, cela prendra typiquement la forme 


\section{LA COMMUNICATION DES NEUROSCIENCES AU TRIBUNAL}

d'une question bien précise adressée à l'expert à laquelle ce dernier devra répondre, devenant ainsi une preuve parmi l'ensemble des faits juridiques accessibles (Jones, Wagner, et al., 2013).

Avant l'engouement pour le neurodroit, certaines preuves expertales en neurosciences étaient déjà sollicitées, telles que l'exposition prénatale à l'alcool, la présence de traumatisme craniocérébral, les tests neuropsychologiques, l'imagerie par résonnance magnétique fonctionnelle (IRMf), l'électroencéphalogramme et la spectroscopie par émission de positons (Chandler, 2016). C'est toutefois l'IRMf qui a propulsé l'avènement du neurodroit, à cause de ses images dynamiques des processus cérébraux (Gkotsi et al., 2015). En effet, l'expert peut présenter des images mettant en lumière des anomalies telles que le dysfonctionnement d'une structure cérébrale ou une lésion cérébrale. Celles-ci sont présentées conjointement à des études empiriques démontrant que ce type d'anomalies est la cause biologique d'un trouble mental pouvant entraver la rationalité, le contrôle du comportement (impulsions) ou les capacités mentales générales (Lamparello, 2011).

Aux États-Unis, le premier cas utilisant l'IRMf en défense contre une peine de mort tentait de démontrer la présence d'un diagnostic de psychopathie en présentant des images d'IRMf, où l'on percevait de faibles activités dans les zones cérébrales responsables de la perception des émotions et de la prise de décisions (amygdale, insula et cortex orbitofrontale; Hughes, 2010). Au Canada, les données neuroscientifiques sont habituellement utilisées par la défense en droit criminel dans le but de nier ou d'amoindrir la responsabilité criminelle de l'accusé qui est soupçonné d'agression sexuelle, de meurtre, d'assaut ou de vol (Chandler, 2016). En parallèle à l'évaluation de la responsabilité criminelle, les données neuroscientifiques sont également utilisées afin de déterminer le niveau de dangerosité de l'accusé (p. ex., le risque de 
récidive criminelle) ainsi que le pronostic de traitement (p. ex., caractère irrémédiable ou non du comportement criminel de l'accusé).

\section{Défis dans la transposition des connaissances du laboratoire au tribunal}

Prises dans leur globalité, les études portant sur l'impact des neurosciences à titre de preuves sont mitigées (p. ex., quant à la valeur accordée aux images par les juges en comparaison à d'autres types de preuves ainsi qu'à leurs impacts juridiques), certaines n'ayant pas trouvé d'impact (Desmoulin-Canselier, 2020; Roskies et al., 2013; Schweitzer et al., 2011), tandis que d'autres oui (Aspinwall et al., 2012; Chandler, 2016; Claeys \& Vialatte, 2012).

Toutefois, cela n'invalide en rien la pertinence d'une réflexion entourant le transfert des connaissances. D'une part, une communication sous-optimale comporte un risque de donner l'illusion d'une validité intrinsèquement supérieure aux neurosciences dépassant la valeur probante réelle des faits invoqués. Un avocat veut convaincre le juge ou le jury, puis il semble que l'usage des neurosciences soit potentiellement un outil pour y parvenir. Certains parlent de «technological sexiness » afin de décrire le caractère implicitement impressionnant et persuasif associé aux neurosciences (Jones, Wagner, et al., 2013, p. 730). Ainsi, lorsqu'un expert fait référence au système nerveux ou que son opinion est basée sur une image cérébrale, sa preuve expertale peut parfois être classée implicitement comme étant scientifique (Ginther, 2016). Les neurosciences impliquent une démarche empirique et rigoureuse qui permet de distinguer un fait scientifique établi d'une opinion, d'une spéculation ou d'une hypothèse. Ainsi, ce n'est pas l'utilisation d'une technologie (p. ex., IRMf) ou un objet de recherche (p. ex., cerveau) qui implique que les résultats obtenus soient scientifiques, mais bien la démarche scientifique (Ginther, 2016). Lors de l'interprétation des résultats et de son utilisation au tribunal, il est donc 
primordial de porter attention à la rigueur de celle-ci afin d'éviter les usages inappropriés ou les exagérations.

D'autre part, la détermination de faits scientifiques n'implique pas nécessairement de causalité. Gkotsi et Gasser (2016) exposent diverses problématiques liées à l'interprétation des résultats considérés « scientifiquement valides ». Par exemple, les résultats de l'IRMf sont habituellement basés sur des différences de moyennes entre un groupe clinique et un groupe contrôle, de manière à ce que l'accent soit mis sur les tendances générales et non sur la pertinence ou l'adéquation pour un cas spécifique. Cette limite associée à l'inférence est aussi valable pour les études corrélationnelles (Mackintosh \& Baddeley, 2011). Aussi, une inférence causale à partir d'une zone cérébrale précise est scientifiquement intenable dans bien des cas (Gkotsi \& Gasser, 2016; Mackintosh \& Baddeley, 2011). En effet, plusieurs régions cérébrales interconnectées sous-tendent habituellement une fonction cognitive, et non une région spécifique. De surcroît, une région spécifique (p. ex., lobe frontal) peut référer à différents processus psychologiques et cognitifs (p. ex., mémoire de travail, planification, prise de décision, etc.). Même dans le cas où le témoignage ne porte pas sur les zones cérébrales, mais plutôt sur un trouble ayant une origine neurologique (p. ex., un trouble du spectre de l'autisme), le passage du diagnostic à la causalité est également difficile (Freckelton, 2012). D’autres auteurs mettent de l'avant que chaque comportement a nécessairement une cause, et donc que la causalité ne devrait pas avoir de répercussions légales (p. ex., Morse, 2006). De ce point de vue, la preuve neuroscientifique serait une explication du comportement de l'individu — non pas un critère pour juger de sa responsabilité criminelle. Un diagnostic ne permettrait ainsi pas d'excuser un acte donné, et ce, d'autant plus sachant que les individus ayant un même diagnostic peuvent avoir des comportements bien hétérogènes (Morse, 2019). Enfin, il est important de considérer 
que le tribunal s'intéresse à l'état mental de l'individu au moment du crime. Les preuves neuroscientifiques ne permettent pas nécessairement de faire des inférences valides par rapport à ce moment précis, ce qui est un élément crucial pour juger de la responsabilité criminelle (Gkotsi \& Gasser, 2016; Mackintosh \& Baddeley, 2011).

\section{Défis du témoignage : l'exemple de la médecine légale}

Le témoignage de l'expert au tribunal peut avoir un impact décisif sur le jugement rendu, mais peut malheureusement faire l'objet de défis majeurs, comme démontré par des exemples provenant de la médecine légale canadienne. En 2008, il y a eu la Commission d'enquête sur la médecine légale pédiatrique en Ontario, qui faisait suite à une série d'erreurs majeures commises lors d'expertises. Bien que cette commission portait sur un ensemble plus large de problèmes judiciaires liés à l'expertise, le rapport du commissaire Goudge (2008) exprimait déjà très clairement des préoccupations quant aux habiletés de communication des experts :

[Le] but premier de la médecine légale est de servir le système de justice. Lorsque les opinions des médecins légistes [ou autres experts], ainsi que leurs limites, ne sont pas bien comprises, le fonctionnement du système de justice prend alors appui sur des bases erronées. Ce problème de communication peut avoir des conséquences graves et parfois même désastreuses sur l'administration de la justice et sur les personnes principalement concernées, dont les accusés et les familles [...]. Les personnes innocentes ne devraient pas être accusées ni reconnues coupables et les personnes coupables ne devraient pas être libérées en se basant sur des opinions d'expert mal interprétées (p. 445).

Ainsi, il y a tout lieu de penser que les enjeux énumérés par le commissaire Goudge sont aussi valables pour l'expertise neuroscientifique, puis de vouloir travailler en amont afin d'éviter que de telles erreurs ne se reproduisent.

\section{Objectif}

Même si les connaissances neuroscientifiques pullulent depuis les dernières années et que leur utilisation au tribunal croît, la communication des neurosciences est un domaine de 
recherche qui demeure à ce jour peu étudié. En effet, si le développement des connaissances théoriques et appliquées est nécessaire en soi, il en va de même pour la capacité à les présenter et à les vulgariser adéquatement aux juges, avocats et jurés. Considérant que les expertises peuvent avoir des implications importantes sur le plan légal et que le témoin expert porte avec lui la crédibilité de sa discipline (p. ex., Direction des enquêtes du Collège des médecins du Québec, 2016), le fait que la communication n'a pas été l'objet de la même attention empirique s'avère préoccupant. En continuité avec les enjeux précédemment énumérés, cet article vise à soutenir la pertinence du développement d'un courant de recherche portant sur la communication des neurosciences par les experts témoignant au tribunal. Il est question de mettre l'accent sur le processus (p. ex., qu'est-ce qui fait qu'un témoignage est adéquatement transmis ?) plutôt que sur le résultat purement juridique (p. ex., est-ce que le témoignage a permis de faire pencher la balance ?). Le processus (p. ex., vulgarisation, nuances apportées) est fortement susceptible d'avoir un impact sur le résultat (p. ex., verdict de culpabilité, sentence), indépendamment du poids réel des faits scientifiques invoqués, c'est pourquoi il est si important de s'y intéresser.

\section{Pourquoi étudier la communication?}

Les experts en neurosciences ont la difficile tâche de lier la science et le droit, puis de s'assurer que leur message soit bien interprété par les acteurs juridiques. Cette tâche va donc de pair avec celle d'éducation (Freckelton, 2012). Cela devient tout particulièrement essentiel dans un contexte où les concepts sont complexes, les termes hautement spécialisés, puis le risque de confusion par l'usage de termes ayant un sens différent selon les disciplines bien réel (p. ex., les termes « preuve » ou « significatif » ont des sens très différents en science et en droit; Jones, Wagner, et al., 2013). La capacité d'un expert à vulgariser et à nuancer ce qui constitue, d'abord, un fait au sens de la science est donc un préalable pour mener à une meilleure détermination de 
ce qui constitue, ensuite, un fait au sens du droit. Bien que la distinction entre un fait scientifique et une spéculation devrait être claire pour les scientifiques (Ginther, 2016), elle est susceptible de l'être beaucoup moins pour les personnes assistant à une audience au tribunal (p. ex., avocats, juges, jurés, etc.). Ainsi, une transmission accessible, cohérente et nuancée du savoir scientifique permet une distinction nette entre les faits et les spéculations au tribunal, et semble nécessaire pour « maintenir la science au sein des neurosciences » (traduction libre; Ginther, 2016, p. 235). Il est donc d'une importance capitale que l'expert sache communiquer de façon juste et efficace afin que ces nuances se reflètent dans la compréhension des auditeurs du tribunal, puis pour éviter tout sensationnalisme. Puisqu'il est plus probable de ne pas bien comprendre les neurosciences que de bien les comprendre, il devient d'autant plus important que les neuroscientifiques prennent à cœur la responsabilité d'un transfert optimal des connaissances (Jones, Wagner, et al., 2013). En effet, nul n’est servi par un «jeu de téléphone arabe » : ni les juges et jurés, qui voient leurs réflexions perturbées par un bruit inutile, ni les experts, qui pourraient malencontreusement être discrédités par leurs difficultés à communiquer, ni les neurosciences au sens large, qui risquent d'être assimilées à la pseudoscience ou à une marchandise.

Les sciences sociales, dont la psychologie, permettent aux professionnels de ce domaine de porter un regard critique sur des thèmes comme la communication efficace, les biais implicites, de même que l'influence des pairs, pouvant ainsi jouer un rôle clé dans l'étude du phénomène. En effet, la recherche portant sur l'amalgame droit-psychologie est bien ancrée depuis des décennies au Canada, tant sur le plan appliqué qu'expérimental, puis rayonne partout dans le monde (p. ex., Dalby, 2014). C'est pourquoi le présent article s'adresse à cette 
communauté et vise à stimuler la recherche sur ces thèmes essentiels. Les implications pouvant découler d'un tel courant de recherche et des devis de recherche associés sont proposés.

\section{Implications et directions futures}

\section{Implications}

À long terme, ce courant de recherche permettrait de promouvoir de meilleures pratiques chez les experts en neurosciences, facilitant ainsi le transfert des connaissances. En consultant des juristes, des experts et des chercheurs dans leur élaboration, ces efforts pourraient se traduire par le développement de lignes directrices spécifiques s'adressant aux experts et à des formations visant la consolidation de différentes compétences. L’identification des lacunes dans le processus de transmission du savoir déterminerait les besoins de formation. En effet, une formation complète à la communication des neurosciences en contexte d'expertise, basée sur une attitude de perfectionnement plutôt que de blâme, profiterait à leur témoignage. Centrées sur des thématiques précises, telles que la vulgarisation, la synthèse ou l'utilisation parcimonieuse et nuancée de support visuel, ces formations rehausseraient ultimement l'utilité des témoignages des neuroscientifiques au sein des tribunaux.

Afin d'illustrer à quoi pourraient ressembler de telles recommandations, voici des exemples plus généraux provenant d'un rapport sur les erreurs judiciaires traitant notamment de l'expertise médicolégale au Canada (Groupe de travail du Comité FPT des chefs des poursuites pénales, 2004) : (a) « chercher à mettre en place une politique d'utilisation d'un certain vocabulaire uniforme qui ne serait pas trompeur et qui rehausserait la compréhension » (recommandation 8, p. 129); (b) adopter un langage qui « [fait] ressortir plus clairement les limites des constatations scientifiques » (recommandation 10, p. 129); (c) permettre que «[1]'expert [...] [puisse] quitter la barre des témoins soucieux de la possibilité que son 
témoignage soit mal interprété ou que les conclusions qui peuvent être tirées ou les limites de ces conclusions soient mal comprises » (recommandation 12, p. 130); et (d) mettre sur pied ou à jour des « programmes structurés de formation continue sur un large éventail de questions :

méthodologie scientifique, protection et contrôle des éléments de preuve, tenue de notes, progrès scientifiques, questions testimoniales, indépendance et impartialité, rédaction de rapports, utilisation de la langue, portée et limites des conclusions, et déontologie » (recommandation 25, p. 135). Enfin, une autre recommandation sur les pratiques à favoriser chez les experts, provenant cette fois-ci de lignes directrices en psychologie (American Psychological Association, 2013), est de (e) « faire tout son possible pour différencier observations, inférences et conclusions » (traduction libre; ligne directrice 11.02, p. 16). Ces recommandations générales sont nécessairement aussi valides pour les neuroscientifiques. Cependant, le développement des connaissances sur la communication des neurosciences spécifiquement permettrait de formuler des recommandations plus précises à leur domaine, tenant notamment compte du haut degré de complexité des concepts invoqués, de même que des caractéristiques et enjeux propres à cette discipline.

\section{Directions futures pour les chercheurs}

\section{Élaboration de devis qualitatifs}

Le développement d'un nouveau courant de recherche devrait passer par une recherche aux devis diversifiés. Dans le domaine des sciences humaines et sociales, le courant qualitatif est très utile. Il l'est, d'une part, par sa flexibilité et son utilité pour soulever les questions importantes, puis, d'autre part, par son angle d'analyse non réductionniste, qui met de l'avant la prise en compte du contexte et du point de vue des acteurs (p. ex., Yilmaz, 2013). Il serait donc primordial de rencontrer des acteurs du domaine juridique, notamment des avocats et des experts en 
neurosciences, pour obtenir leur avis sur leurs expériences amalgamant les neurosciences et le droit. Reposant par exemple sur une entrevue semi-structurée, des questions sur des thèmes comme la réceptivité du matériel neuroscientifique, les attentes mutuelles ou, encore, les défis communicationnels des experts, pourraient leur être adressées. Plusieurs avantages pourraient découler d'une telle étude, dont la compréhension de multiples réalités (p. ex., accusé, avocat, juge), la description de phénomènes complexes (p. ex., différences épistémologiques), la prise en compte du contexte des individus (tribunal) et la comparaison d'avis de différents acteurs sur une même problématique.

Les verbatims (notes sténographiques) des procès pourraient également être utilisés pour en analyser le contenu. Plus précisément, les habiletés de vulgarisation des experts pourraient être étudiées en analysant la quantité de mots jargonneux propres aux neurosciences (p. ex., termes inaccessibles et non définis). De plus, l'analyse de contenu thématique pourrait mettre en lumière divers thèmes liés aux neurosciences qui sont plus fréquemment communiqués au tribunal, ainsi que leur impact sur le processus décisionnel.

\section{Élaboration de devis quantitatifs}

La recherche quantitative, pour sa part, permet de fournir des réponses à des questions précises, d'attribuer la causalité à des facteurs définis et d'effectuer des inférences (Yilmaz, 2013). D'abord, il serait important d'étudier l'effet des heuristiques de jugement sur la prise de décision. Ce type d'heuristique consiste en une opération mentale rapide et intuitive utilisée lorsque l'environnement contraint à prendre une décision, typiquement dans un court délai (Koehler et al., 2002). Ils permettent de réduire l'effort cognitif en négligeant certains détails, ce qui mène malheureusement parfois à des erreurs de raisonnement ou à des simplifications. Selon une étude classique (Nisbett et al., 1981), le type d'information présenté (type trivial ou 


\section{LA COMMUNICATION DES NEUROSCIENCES AU TRIBUNAL}

stéréotypé) influence l'utilisation d'heuristiques de jugement, modifiant ainsi la prise de décision. Cette étude pourrait être répliquée en comparant l'influence du type de témoignage sur le juge ou les jurés selon l'utilisation de support visuel. Les participants seraient aléatoirement affectés à un groupe, soit à un groupe expérimental écoutant un expert témoignant avec support visuel (p. ex., numérisations d'IRMf) ou à un groupe contrôle écoutant le même expert qui présente les mêmes résultats sans support visuel. Les participants donneraient ensuite leur verdict, en plus d'évaluer le niveau de crédibilité de l'expert. Afin de contrôler certaines variables confondantes (p. ex., crédibilité et assurance de l'expert, émotion véhiculée, etc.), le témoignage de l'expert pourrait être visionné par les participants en contenu vidéo. La détermination de la causalité n'est pas réalisable à partir de l'étude de décisions judiciaires, où un plan expérimental est impossible à mettre en place (p. ex., comme dans l'étude de DesmoulinsCanselier, 2020). Des études expérimentales permettraient donc d'observer, de façon causale, l'influence des témoignages en neurosciences ainsi que l'impact du support visuel sur les processus décisionnels.

Une autre expérience pourrait viser à déterminer le degré de compréhension des juges et jurés par l'utilisation de témoignages simulés. Les participants pourraient répondre à un questionnaire à la suite d'un témoignage d'expert en neurosciences afin d'évaluer leur niveau de compréhension vis-à-vis de ce qui a été présenté. Cela permettrait de quantifier l'information assimilée et comprise, puis permettrait par le fait même de préciser la nature des lacunes (p. ex., les participants pourraient rapporter un lien de causalité, qui n'a pourtant jamais été énoncé par l'expert). Cette expérience pourrait également être menée en deux temps, où l'expert recevrait une formation de vulgarisation des faits neuroscientifiques entre les deux temps de mesures. Ainsi, il serait possible de créer deux groupes, soit un groupe expérimental et un groupe contrôle. 
Le groupe expérimental visionnerait une vidéo d'un témoignage effectué par un expert avant que celui-ci ne reçoive une formation à la vulgarisation. Dans un deuxième temps, ce groupe visionnerait un témoignage portant sur la même information, mais après que l'expert ait reçu une formation. Le groupe contrôle visionnerait le même extrait vidéo à deux reprises, c'est-à-dire sans que l'expert ait reçu de formation. Les deux groupes répondraient au même questionnaire à chaque temps de mesure. Il serait ainsi possible de mesurer l'impact de la formation qu'a reçu l'expert sur la compréhension des participants.

\section{Conclusion}

Les neurosciences se développent à une grande vitesse depuis les dernières années, puis elles sont en utilisation croissante devant les tribunaux (Chandler, 2016). Les applications dans lesquelles elles pourraient être invoquées semblent nombreuses et inspirent de grands espoirs chez certains acteurs juridiques. Néanmoins, certaines mauvaises expériences judiciaires, basées sur des expertises médicolégales, incitent à vouloir tirer d'importantes leçons du passé. La communication des neurosciences au tribunal est un courant de recherche peu développé. Il est question de déterminer ce qui contribue à la vulgarisation et au maintien de la justesse d'une information qui serait transposée du laboratoire à une salle d'audience. Cela permettrait d'assurer une meilleure compréhension des avocats, juges et jurés, puis de favoriser une utilisation respectant le statut actuel des connaissances. Certaines lignes directrices spécifiques, de même que des formations destinées à améliorer les habiletés communicationnelles des experts, pourraient découler d'un tel courant de recherche. Ainsi, un transfert optimal des connaissances vers les tribunaux serait favorisé, maximisant ainsi leur utilité dans la procédure judiciaire. 


\section{LA COMMUNICATION DES NEUROSCIENCES AU TRIBUNAL}

\section{Références}

American Psychological Association. (2013). Specialty guidelines for forensic psychology. American Psychologist, 68(1), 7-19. https://doi.org/10.1037/a0029889

Aspinwall, L. G., Brown, T. R., \& Tabery, J. (2012). The double-edged sword: Does biomechanism increase or decrease judges' sentencing of psychopaths? Science, 337(6096), 846-849. https://doi.org/10.1126/science.1219569

Barreau du Québec. (2017). Guide des meilleures pratiques (6éd.). https://www.barreau.qc.ca/media/1309/bdq-guide-meilleures-pratiques.pdf

Brunet, L. (2014). Avant-propos — L'expertise psycholégale : balises méthodologiques et déontologiques [Foreword—Expert psychological testimony: Methodological and deontological beacons]. In L. Brunet, L'expertise psycholégale (2e éd., pp. i-vi). Presses de l’Université du Québec.

Bureau du syndic et Comité d'inspection professionnelle de l'Ordre des psychologues du Québec. (2002). L'expertise psycholégale (partie 1). Fiche déontologique, 3(4). https://www.ordrepsy.qc.ca/documents/26707/63191/L\%27expertise+psychol\%C3\%A9g ale+\%28partie+1\%29/c18af058-1b7d-408e-988f-88e23342c605

Chandler, J. A. (2016). The use of neuroscientific evidence in Canadian criminal proceedings. Journal of Law and the Biosciences, 2(3), 550-579. https://doi.org/10.1093/jlb/lsv026

Claeys, A., \& Vialatte, J. (2012). L'impact et les enjeux des nouvelles technologies d'exploration et de thérapie du cerveau (rapport $\mathrm{n}^{\circ}$ 476) [Impact and stakes of new brain exploration and therapy technologies (Report No. 476)]. Office parlementaire d'évaluation des choix scientifiques et technologiques de la France. http://www.assembleenationale.fr/13/pdf/rap-off/i4469.pdf 


\section{LA COMMUNICATION DES NEUROSCIENCES AU TRIBUNAL}

Dalby, J. T. (2014). Forensic psychology in Canada a century after Münsterberg. Canadian Psychology/Psychologie canadienne, 55(1), 27-33. https://doi.org/10.1037/a0035526

Daubert v. Merrell Dow Pharmaceuticals, Inc. [1993], 509 US 579.

Desmoulin-Canselier, S. (2020). Another perspective on "neurolaw": the use of brain imaging in civil litigation regarding mental competence. In A. D’Aolia \& M. C. Errigo, Neuroscience and Law (pp. 529-547). Springer. https://doi.org/10.1007/978-3-030$38840-9$

Direction des enquêtes du Collège des médecins du Québec. (2016). Le mandat d'expertise : transparence et pertinence [Forensic assessment mandate: Transparency and relevance]. Collège des médecins du Québec. http://www.cmq.org/nouvelles-pdf/n-4-2016-01-20-frmandat-expertise-transparence-et-pertinence.pdf?t=1585835426105

Freckelton, I. (2012). Expert evidence by mental health professionals: The communication challenge posed by evidence about autism spectrum disorder, brain injuries, and Huntington's Disease. International Journal of Law \& Psychiatry, 35(5/6), 372-379. https://doi.org/10.1016/j.ijlp.2012.09.008

Ginther, M. (2016). Neuroscience or neurospeculation? Peer commentary on four articles examining the prevalence of neuroscience in criminal cases around the world. Journal of Law and the Biosciences, 3(2), 324-329. https://doi.org/10.1093/jlb/lsw030

Gkotsi, G. M., \& Gasser, J. (2016). Critique de l'utilisation des neurosciences dans les expertises psychiatriques : le cas de la responsabilité pénale [Critique of the use of neuroscience in forensic psychiatric assessments: The case of criminal responsibility]. L'Évolution psychiatrique, 81(2), 434-445. https://doi.org/10.1016/j.evopsy.2015.10.002 


\section{LA COMMUNICATION DES NEUROSCIENCES AU TRIBUNAL}

Gkotsi, G. M., Moulin, V., \& Gasser, J. (2015). Les neurosciences au tribunal : de la responsabilité à la dangerosité. Enjeux éthiques soulevés par la nouvelle loi française [Neuroscience in the courtroom: From responsibility to dangerousness, ethical issues raised by the new French law]. L'Encéphale, 41(5), 385-393. http://dx.doi.org/10.1016/j.encep.2014.08.014

Goudge, S. T. (2008). Commission d'enquête sur la médecine pédiatrique en Ontario. Rapport : Vol. 3. Politique et recommandations. Ministère du Procureur général de l'Ontario. https://www.attorneygeneral.jus.gov.on.ca/inquiries/goudge/fr/report/v3_fr_pdf/Vol_3_Fr .$p d f$

Groupe de travail du Comité FPT des chefs des poursuites pénales. (2004). Rapport sur la prévention des erreurs judiciaires (rapport) [Report on the prevention of miscarriages of justice (report)]. Ministère de la Justice du Canada. https://www.justice.gc.ca/fra/pr-rp/jpcj/rc-ccr/pej-pmj/pej-pmj.pdf

Hughes, V. (2010). Science in court: Head case. Nature, 464(7287), 340-342. https://doi.org/10.1038/464340a

Illes, J., Moser, M. A., McCormick, J. B., Racine, E., Blakeslee, S., Caplan, A., Hayden, E. C., Ingram, J., Lohwater, T., McKnight, P., Nicholson, C., Phillips, A., Sauvé, K. D., Snell, E., \& Weiss, S. (2010). Neurotalk: Improving the communication of neuroscience research. Nature Reviews Neuroscience, 11(1), 61-69. https://doi.org/10.1038/nrn2773

Jones, O. D., Marois, R., Farah, M. J., \& Greely, H. T. (2013). Law and neuroscience. Journal of Neuroscience, 33(45), 17624-17630. http://dx.doi.org/10.1523/JNEUROSCI.325413.2013 
Jones, O. D., \& Shen, F. X. (2012). Law and neuroscience in the United States. In

T. M. Spranger (Ed.), International Neurolaw (pp. 349-380). Springer. http://dx.doi.org/10.1007/978-3-642-21541-4_19

Jones, O. D., Wagner, A. D., Faigman, D. L., \& Raichle, M. E. (2013). Neuroscientists in court. Nature Reviews Neuroscience, 14(10), 730-736. http://dx.doi.org/10.1038/nrn3585

Koehler, D. J., Brenner, L., \& Griffin, D. (2002). The calibration of expert judgment: Heuristics and biases beyond the laboratory. In T. Gilovich, D. Griffin, \& D. Kahneman (Eds.), Heuristics and Biases: The Psychology of Intuitive Judgment (pp. 686-715). Cambridge University Press.

Lamparello, A. (2011). Using cognitive neuroscience to predict future dangerousness. Columbia Human Rights Law Review, 42(2), 481-539.

https://papers.ssrn.com/sol3/papers.cfm?abstract_id=1742940

Mackintosh, N., \& Baddeley, A. (2011). Brain waves module 4: Vol. 35. Neuroscience and the law. Royal Society.

McCabe, D. P., \& Castel, A. D. (2008). Seeing is believing: The effect of brain images on judgments of scientific reasoning. Cognition, 107(1), 343-352. http://dx.doi.org/10.1016/j.cognition.2007.07.017

Morse, S. J. (2006). Brain overclaim syndrome and criminal responsibility: A diagnostic note. Ohio State Journal of Criminal Law, 3(2), 397-412. https://repository.upenn.edu/cgi/viewcontent.cgi?article=1027\&context=neuroethics_pub 
Morse S. J. (2019). Neuroscience and criminal law: Perils and promises. In L. Alexander \& K. K. Ferzan (Eds), The Palgrave handbook of applied ethics and the criminal law (pp. 471-496). Palgrave Macmillan. https://doi.org/10.1007/978-3-030-22811-8_20

Nisbett, R. E., Zukier, H., \& Lemley, R. E. (1981). The dilution effect: Nondiagnostic information weakens the implications of diagnostic information. Cognitive Psychology, 13(2), 248-277. https://doi.org/10.1016/0010-0285(81)90010-4

R. v. Mohan [1994], 89 CCC (3d) 402 (CSC).

Roskies, A. L., Schweitzer, N. J., \& Saks, M. J. (2013). Neuroimages in court: Less biasing than feared. Trends in Cognitive Sciences, 17(3), 99-101. https://doi.org/10.1016/j.tics.2013.01.008

Schweitzer, N. J., Saks, M. J., Murphy, E. R., Roskies, A. L., Sinnott-Armstrong, W., \& Gaudet, L. M. (2011). Neuroimages as evidence in a mens rea defense: No impact. Psychology, Public Policy, and Law, 17(3), 357-393. https://doi.org/10.1037/a0023581

Service des poursuites pénales du Canada. (2011). Un système plus juste : la voie vers l'élimination des condamnations injustifiées (rapport) [The path to justice: Preventing wrongful convictions (report)]. https://www.ppsc-sppc.gc.ca/fra/pub/spj-ptj/ch9.html

Stettler, G. (2019). L'administration de la preuve scientifique en droit nord-américain [The administration of scientific evidence in North American law]. Canadian Bar Review/Revue du Barreau canadien, 97(1), 177-239. https://cbr.cba.org/index.php/cbr/article/view/4512

Yilmaz, K. (2013). Comparison of quantitative and qualitative research traditions: Epistemological, theoretical, and methodological differences. European Journal of Education, 48(2), 311-325. https://doi.org/10.1111/ejed.12014 